Check for updates

Cite this: RSC Adv., 2020, 10, 19211

\title{
Analytical and preparative separation and isolation of functionalized fullerenes by conventional HPLC stationary phases: method development and column screening $\dagger$
}

\begin{abstract}
Merve Ergun Dönmez (iD and Helena Grennberg (iD *
Isolation and purification of functionalized fullerenes from often complex reaction mixtures is challenging due to the hydrophobic nature and low solubility in regular organic solvents. We have developed an HPLC method that efficiently, employing regular reversed phase stationary phases, separates not only $\mathrm{C}_{60}$ from $\mathrm{C}_{70}$ in a model mixture, but also $\mathrm{C}_{60}$ monoadducts from polyadducts and unreacted $\mathrm{C}_{60}$ from fulleropyrrolidine and hydroarylation example reaction mixtures. Six HPLC columns with regular reversed phase stationary phases were evaluated using varying proportions of acetonitrile in toluene as eluent; with C18 and C12 stationary phases with high surface area $\left(450-400 \mathrm{~m}^{2} \mathrm{~g}^{-1}\right)$ being the most efficient regarding separation efficiency and analysis time for all mixtures. The analytical method is effectively transferrable to a preparative scale to isolate the monoaddition products from complex fullerene reaction mixtures.
\end{abstract}

Received 27th March 2020

Accepted 8th May 2020

DOI: 10.1039/d0ra02814b

rsc.li/rsc-advances analytical HPLC applications. ${ }^{28-30}$ However, a more generally applicable method for analysis and separation of complex fullerene mixtures using regular HPLC columns and standard solvents has been lacking. Herein, we report a method that give efficient analytical and preparative separation using standard stationary phases and toluene-acetonitrile mobile phases for model mixtures of $\mathrm{C}_{60}$ and $\mathrm{C}_{70}$ as well as for two example reaction mixtures: pyrrolidination ${ }^{31}$ and hydroarylation, ${ }^{32}$ (Scheme 1) reactions that result in different proportions of unreacted $\mathrm{C}_{60}$, monoadducts and higher adducts.

planar aromatic compounds where a new substituent affect the reactivity even at remote $\pi$-conjugated positions, fullerenes react at multiple positions leading to complex hardto-separate mixtures of mono-, bis- and more highly functionalized fullerenes. ${ }^{16-19}$ Due to poor solubility in most solvents, purification of product mixtures with isolation of the desired components and assessment of purity are major challenges. $^{20-23}$

Already in 1990, Kroto et al. ${ }^{24}$ identified liquid chromatography (LC) as a purification method that could provide $\mathrm{C}_{60}$ and $\mathrm{C}_{70}$ as clean fractions from their complex synthesis soot mixtures. More recently, chromatographic separations on silica and alumina have been reported, however employing solvents with poor environmental profile. ${ }^{25-27}$ Alternatives, in particular the fullerene-specific stationary phases, opened for LC methods that employ less harmful solvents for both preparative and

Uppsala University, Department of Chemistry - BMC, Box 576, 75123 Uppsala, Sweden.E-mail: helena.grennberg@kemi.uu.se

$\dagger$ Electronic supplementary information (ESI) available. See DOI: 10.1039/d0ra02814b

\section{Materials and methods}

\subsection{Reagents and materials}

All chemicals, including $\mathrm{C}_{60}$ and $\mathrm{C}_{70}$ were purchased from Sigma Aldrich and used as received. Acetonitrile and toluene was purchased from VWR Chemicals as HPLC grade. The columns used are listed in Table 1 . The analytical columns were used with a Gemini C18 pre-guard cartridge $(2.0 \times 4.0 \mathrm{~mm}$, Phenomenex, USA), the preparative column was used with ACE 5 C18 (10.0 × 10.0, Advanced Chromatography Technologies Ltd, UK) pre-guard cartridge.

In order to describe the quality of the peaks and the efficiency of the separation for all analytical columns, retention times $\left(R_{\mathrm{t}}\right)$, total analysis time (run time), capacity factors $\left(k^{\prime}\right)$ and resolution $\left(R_{\mathrm{S}}\right)$ were evaluated and summarized in Tables 2 and 3 . The capacity factor $\left(k^{\prime}\right)$ is a measure that gives the molecules retention compared to the un-retained component or solvent, typically preferred to be higher than 0.8 . Resolution $\left(R_{\mathrm{s}}\right)$ is 


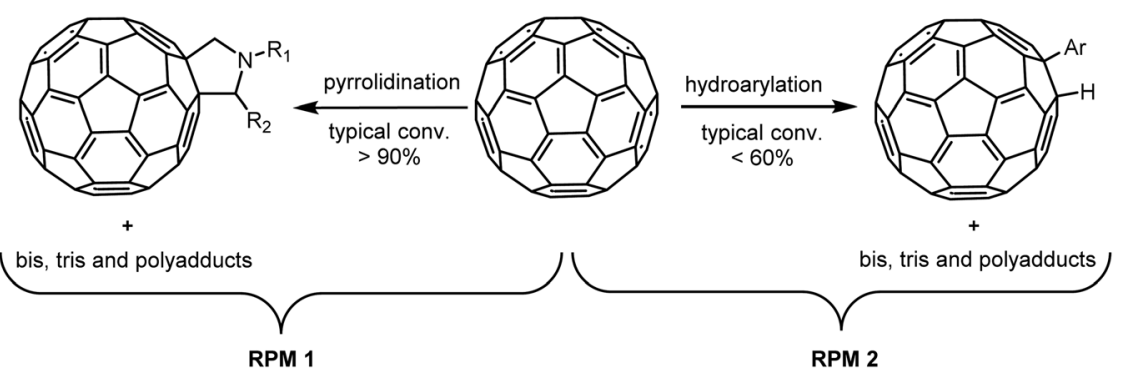

Scheme 1 General representation of pyrrolidination and hydroarylation of $C_{60}$ by literature conditions, resulting in the two reaction product mixtures of the present study.

Table 1 The coding and properties of the columns used in this study

\begin{tabular}{|c|c|c|c|c|c|c|c|}
\hline $\begin{array}{l}\text { Column } \\
\text { code }\end{array}$ & Brand name & $\begin{array}{l}\text { Inner dimensions } \\
\text { length } \times \text { radius }\end{array}$ & $\begin{array}{l}\text { Particle } \\
\text { size }\end{array}$ & $\begin{array}{l}\text { Surface area } \\
\left(\mathrm{m}^{2} \mathrm{~g}^{-1}\right)\end{array}$ & $\begin{array}{l}\text { Carbon load } \\
(\%)\end{array}$ & Column packing & End-capping \\
\hline $\operatorname{Col} 1^{a}$ & Inertsil ODS 4 & $250 \times 4.6 \mathrm{~mm}$ & $5.0 \mu \mathrm{m}$ & 450 & 11 & $\mathrm{C}_{18}$ & TMS \\
\hline $\operatorname{Col~} 3^{b}$ & Synergi Max-RP & $150 \times 4.6 \mathrm{~mm}$ & $4.0 \mu \mathrm{m}$ & 400 & 17 & $\mathrm{C}_{12}$ & TMS \\
\hline Col $4^{b}$ & Gemini NX & $100 \times 3.0 \mathrm{~mm}$ & $3.0 \mu \mathrm{m}$ & 375 & 14 & $\mathrm{C}_{18}$ & TMS \\
\hline Col $5^{b}$ & Kinetex Biphenyl & $100 \times 4.6 \mathrm{~mm}$ & $2.6 \mu \mathrm{m}$ & 200 & 11 & $\mathrm{C}_{12}$ (biphenyl) & TMS \\
\hline
\end{tabular}

${ }^{a}$ From GL Sciences, Japan. ${ }^{b}$ From Phenomenex, USA. ${ }^{c}$ From Advanced Chromatography Technologies Ltd, UK.

a measure to describe the separation between two adjacent peaks, typically preferred to be higher than 2.0 .

\subsection{Instrumentation}

All analyses were carried out using a Gilson HPLC system consisting of a Gilson 331 Pump, Gilson 156 Dual UV/VIS detector, Gilson 215 Nebula Liquid Handler and fraction collector, Gilson 819 Injection Module and Gilson 506C System Interface, using Gilson Unipoint Software (version 5.11, USA). The HPLC separations were performed using isocratic flow rate and the columns were kept at room temperature (between $20^{\circ} \mathrm{C}$ and 23 ${ }^{\circ} \mathrm{C}$ ). Detection was carried out at $285 \mathrm{~nm}$ and $350 \mathrm{~nm}$ simultaneously. In order to monitor the mobile phase effects, four different mobile phases; toluene, $45 \%(\mathrm{v} / \mathrm{v})$ acetonitrile in toluene, $50 \%(\mathrm{v} / \mathrm{v})$ acetonitrile in toluene and $55 \%(\mathrm{v} / \mathrm{v})$ acetonitrile in toluene were used. The flow rate was adjusted between 0.4-1 $\mathrm{mL} \mathrm{min}^{-1}$ based on the backpressure of the columns. Injection volumes were between $30-50 \mu \mathrm{L}$ for analytical separation, and 4000-5000 $\mu \mathrm{L}$ for preparative separation. Preparative separations were conducted by applying the corresponding analytical methodology but increasing the injection volume and

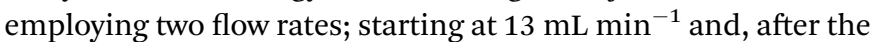
elution of monoadduct, increased to $16 \mathrm{~mL} \mathrm{m^{-1 }}$ for faster elution of unreacted $\mathrm{C}_{60}$. All the injections were made within 30 minutes of the sample preparation.

NMR spectra of reaction mixtures and isolated monoadducts were recorded on a Varian $500 \mathrm{MHz}$ and Agilent $400 \mathrm{MHz}$ NMR Spectrometers. Mass spectrometry of reaction mixtures and isolated monoadducts was carried out using a Bruker Autoflex 2
MALDI-TOF spectrometer in positive and negative ion mode and trans-2-[3-(4-tert-butylphenyl)-2-methyl-2-propenylidene] malononitrile (DCTB) was used as matrix. Matrix solution was prepared by dissolving $10 \mathrm{mg}$ of DCTB in $1 \mathrm{~mL}$ of chloroform. The analytes to be measured were then dissolved in chloroform and mixed with a small amount of matrix solution and loaded on the target plate.

\subsection{Sample preparation for HPLC analyses}

2.3.1. $\mathrm{C}_{60}+\mathrm{C}_{70}$ mixture solution that was prepared from $\mathrm{C}_{60}$ and $\mathrm{C}_{70}$ solutions

2.3.1.1. $C_{60}$ solution. $15 \mathrm{mg}$ of $\mathrm{C}_{60}$ was weighed to a $10 \mathrm{~mL}$ volumetric flask and dissolved using toluene by sonication and marked up to its volume by toluene. Then $4 \mathrm{~mL}$ of this stock solution was pipetted into a $10 \mathrm{~mL}$ volumetric flask and diluted to its volume by $50 \%(\mathrm{v} / \mathrm{v})$ toluene in acetonitrile or toluene depending on the mobile phase. The solution is then mixed by shaking thoroughly and filtered through $0.45 \mu \mathrm{m}$ PVDC filter into an HPLC vial.

2.3.1.2. $C_{70}$ solution. $7 \mathrm{mg}$ of $\mathrm{C}_{70}$ was weighed to a $5 \mathrm{~mL}$ volumetric flask and dissolved using toluene by sonication and marked up to its volume by toluene. Then $2 \mathrm{~mL}$ of this stock solution was pipetted into a $10 \mathrm{~mL}$ volumetric flask and diluted to its volume by $50 \%(\mathrm{v} / \mathrm{v})$ toluene in acetonitrile or toluene depending on the mobile phase. The solution is then mixed by shaking thoroughly and filtered through $0.45 \mu \mathrm{m}$ PVDC filter into an HPLC vial.

2.3.1.3. $C_{60}+C_{70}$ mixture solution. $2 \mathrm{~mL}$ of $\mathrm{C}_{60}$ solution and $2 \mathrm{~mL}$ of $\mathrm{C}_{70}$ solution were pipetted into a $10 \mathrm{~mL}$ volumetric flask 
and diluted to its volume using $50 \%(\mathrm{v} / \mathrm{v})$ toluene in acetonitrile. The solution is then mixed by shaking thoroughly and filtered through $0.45 \mu \mathrm{m}$ PVDC filter into an HPLC vial.

2.3.2. Reaction product mixture 1 (RPM1). An in-house version of fulleropyrrolidine formation reaction reported by Prato et $a l^{31}$ was applied using $\mathrm{C}_{60}$, sarcosine and $p$-anisaldehyde (detailed procedure as well as NMR and MS analyses in ESI $\dagger$ ). After the reaction, pre-purification was performed to wash away the unreacted reagents yielding a brown residue (RPM1) containing only the unreacted fullerenes and fullerene adducts.

For analytical HPLC sample preparation, approximately $15 \mathrm{mg}$ of RPM1 was weighed to a $10 \mathrm{~mL}$ volumetric flask and dissolved using toluene by sonication and marked up to its volume by toluene. Then $4 \mathrm{~mL}$ of this solution was pipetted into a $10 \mathrm{~mL}$ volumetric flask and diluted to its volume by $50 \%(\mathrm{v} / \mathrm{v})$ toluene in acetonitrile or toluene depending on the mobile phase. The solution is then mixed by shaking thoroughly and filtered through $0.45 \mu \mathrm{m}$ PVDC filter into an HPLC vial.

For preparative HPLC sample preparation, approximately $12 \mathrm{mg}$ of RPM1 was weighed to a $10 \mathrm{~mL}$ volumetric flask and dissolved using $6 \mathrm{~mL}$ of toluene by sonication. Then the solution is diluted to its volume with acetonitrile, mixed by shaking thoroughly and sonicated for 10 minutes. The solution is then centrifuged for 5 minutes at $3000 \mathrm{rpm}$.

2.3.3. Reaction product mixture 2 (RPM2). An in-house version of the rhodium-catalyzed hydroarylation reaction reported by Itami et al. ${ }^{32}$ was applied using $\mathrm{C}_{60}, p$-tolylboronic acid with $\left[\mathrm{Rh}(\mathrm{cod})(\mathrm{MeCN})_{2}\right] \mathrm{BF}_{4}$ as catalyst (detailed procedure as well as NMR and MS analyses in ESI $\dagger$ ). After the reaction, prepurification was performed to wash away catalyst and unreacted reagents yielding a brown residue (RPM2) containing only the unreacted fullerenes and fullerene adducts.

For analytical HPLC sample preparation, approximately $15 \mathrm{mg}$ of RPM2 was weighed to a $10 \mathrm{~mL}$ volumetric flask and dissolved using toluene by sonication and marked up to its volume by toluene. Then $4 \mathrm{~mL}$ of this solution was pipetted into a $10 \mathrm{~mL}$ volumetric flask and diluted to its volume by $50 \%(\mathrm{v} / \mathrm{v})$ toluene in acetonitrile or toluene depending on the mobile phase. The solution is then mixed by shaking thoroughly and filtered through $0.45 \mu \mathrm{m}$ PVDC filter into an HPLC vial.

For preparative HPLC sample preparation, approximately $12 \mathrm{mg}$ of RPM2 was weighed to a $10 \mathrm{~mL}$ volumetric flask and dissolved using $6 \mathrm{~mL}$ of toluene by sonication. Then the solution is diluted to its volume with acetonitrile, mixed by shaking thoroughly and sonicated for 10 minutes. The solution is then centrifuged for 5 minutes at $3000 \mathrm{rpm}$.

\section{Results and discussion}

\subsection{Fullerene separation by different mobile phases and columns}

To understand the response of different column packings to fullerenes, $\mathrm{C}_{60}+\mathrm{C}_{70}$ mixture solution was injected to all columns using toluene as a mobile phase. None of the columns showed any retention of the fullerenes as the molecular interactions of fullerenes with the stationary phase are much weaker than the solubilizing interactions of fullerenes with toluene. $\mathrm{C}_{60}$ and $\mathrm{C}_{70}$ were eluted together without any separation. Adding acetonitrile, a very convenient solvent for HPLC analyses ${ }^{20}$ in which fullerenes are very poorly soluble as co-eluent decreased the solubilizing strength of the eluent and resulted in improved separation.

The mobile phase compositions were scanned by injecting $\mathrm{C}_{60}+\mathrm{C}_{70}$ mixture solution using Col 1 (Fig. 1). At $25 \%(\mathrm{v} / \mathrm{v})$ acetonitrile in toluene as mobile phase composition, $\mathrm{C}_{70}$ peak started to get separated from $\mathrm{C}_{60}$ peak and with $35 \%(\mathrm{v} / \mathrm{v})$ acetonitrile in toluene, the peaks resolved completely with a run time of 10 minutes. The chromatogram shows that this mobile phase composition holds great potential for separating higher fullerene compounds which will elute later than $\mathrm{C}_{60}$ and $\mathrm{C}_{70}$ (ref. 33 and 34) within a reasonably short run time. A gradient method can be developed to elute higher fullerenes by gradually decreasing the amount of acetonitrile in the eluent or using an additional co-eluent in which fullerenes are more soluble such as chlorobenzene or carbon disulfide. On the other

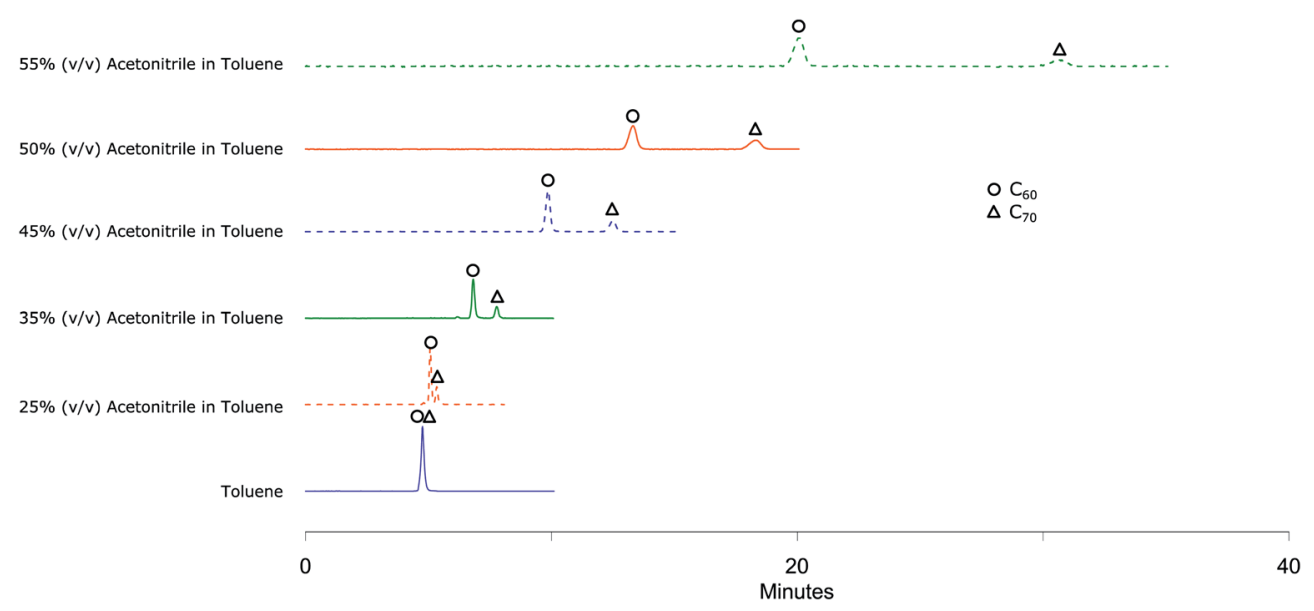

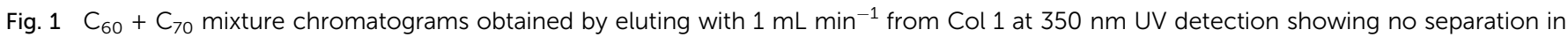
toluene, moderate separation in $25 \%(\mathrm{v} / \mathrm{v})$ acetonitrile in toluene, very good separation in all other mobile phase compositions. 

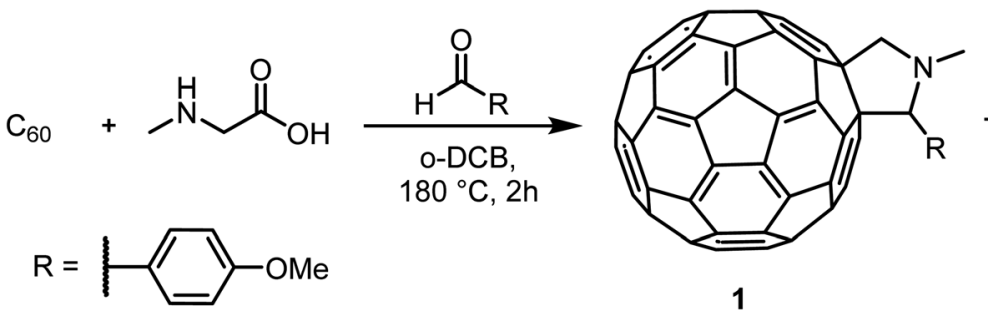

bis, tris

polyadducts

1

Scheme 2 Pyrrolidination/1,3-dipolar cycloaddition reaction of fullerene $\mathrm{C}_{60}$ with 4-anisaldehyde and sarcosine. Before HPLC sample preparation, all reagents were washed away by extraction, having only unreacted $\mathrm{C}_{60}$, product 1 , bis, tris and higher adducts in the RPM1.

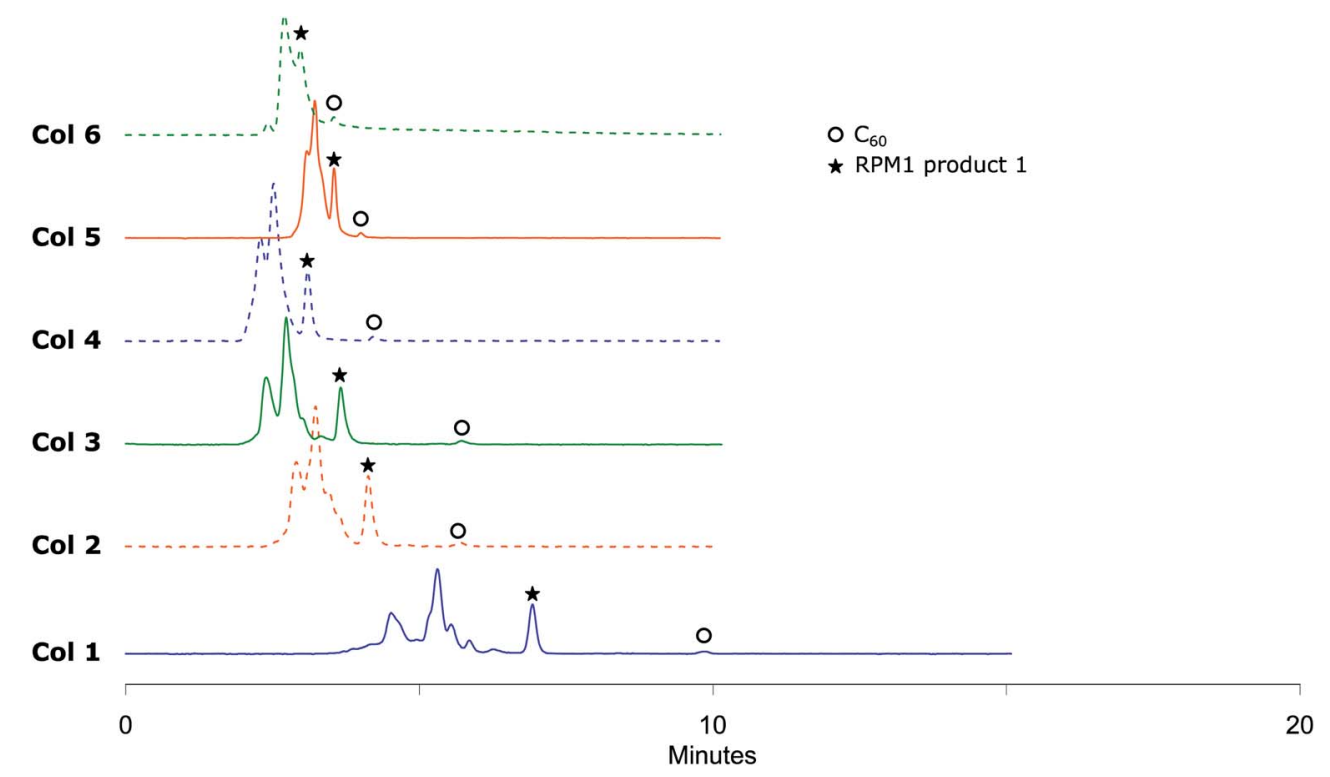

Fig. 2 RPM1 chromatograms obtained by eluting with 45\% (v/v) acetonitrile in toluene at $285 \mathrm{~nm}$ UV detection where Col 1 , Col 2 and Col 3 show very good separation in a short analysis time, Col 4 shows moderate separation and Col 5 and Col 6 does not separate RPM1 product 1 from bis, tris and polyadducts and almost no separation from unreacted $C_{60}$.

hand, since our focus was on separation of functionalized fullerenes which are expected to be eluted faster than unreacted fullerenes ${ }^{35-39}$ and on developing a method with better solvents, higher retention rather than lower is required. This was obtained by increasing the proportion of acetonitrile to $45 \%(\mathrm{v} / \mathrm{v})$ in toluene or higher.

\subsection{Analysis of reaction product mixture 1 (RPM1)}

Fulleropyrrolidination (Prato reaction) ${ }^{31}$ is one of the most wellstudied reactions for functionalizing fullerenes. The reaction usually results in near complete conversion of the starting material to a mixture of different products including mono-, diand poly-adducts. Several separation systems are reported in the literature but most are either relying on $\mathrm{CS}_{2}$ as chromatography solvent or are built around special stationary phases. Encouraged by the efficient separation of $\mathrm{C}_{60}$ and $\mathrm{C}_{70}$ in regular stationary phases we applied the method on fullerene fulleropyrrolidination reaction mixtures (RPM 1) prepared in house (Scheme 2).

Solutions of RPM1 were injected to all six analytical columns using $45 \%, 50 \%$ and $55 \%(\mathrm{v} / \mathrm{v})$ acetonitrile in toluene as eluent.
According to the recorded chromatograms using $45 \%(\mathrm{v} / \mathrm{v})$ acetonitrile in toluene as mobile phase (Fig. 2), $\mathrm{Col} 1$, Col 2 and $\mathrm{Col} 3$ showed very good resolution between $\mathbf{1}$ and unreacted $\mathrm{C}_{60}$ as well as the poly-addition products, with a short run time of 10 minutes for $\mathrm{Col} 2$ and $\mathrm{Col} \mathrm{3.} \mathrm{Col} 4$ showed moderate separation and Col 5 and Col 6 were unable to separate the product with this mobile phase (Table 2). However, Resolution

Table 2 Peak properties of $\mathrm{C}_{60}$ and product 1 on different columns using $45(\mathrm{v} / \mathrm{v})$ acetonitrile in toluene as mobile phase where $R_{\mathrm{t}}$ is retention time, $k^{\prime}$ is capacity factor and $R_{\mathrm{s}}$ is resolution

\begin{tabular}{llllll}
\hline & $R_{\mathrm{t}}\left(\mathrm{C}_{60}\right)$ & $R_{\mathrm{t}}(\mathbf{1})$ & Run time & $k^{\prime}(\mathbf{1})$ & $R_{\mathrm{s}}{ }^{a}$ \\
\hline Col 1 & 9.9 & 6.9 & 13 & 0.8 & 2.2 \\
Col 2 & 5.7 & 3.7 & 8 & 0.8 & 3.2 \\
Col 3 & 5.7 & 4.1 & 8 & $\boldsymbol{0 . 5}$ & 2.2 \\
Col 4 & 4.2 & 3.1 & 7 & $\boldsymbol{0 . 5}$ & $\mathbf{1 . 8}$ \\
Col 5 & 3.5 & 3.0 & 6 & $\boldsymbol{O . 3}$ & $\boldsymbol{N}$ \\
Col 6 & 4.0 & 3.6 & 7 & $\boldsymbol{0 . 2}$ & $\boldsymbol{N}$
\end{tabular}

${ }^{a}$ Calculated according to the closest peak. NS: not separated. The values in bold/italic are outside the ideal range. 
Table 3 Peak properties of 2 and $C_{60}$ on different columns using 45\% $(\mathrm{v} / \mathrm{v})$ acetonitrile in toluene as mobile phase where $R_{\mathrm{t}}$ is retention time, $k^{\prime}$ is capacity factor and $R_{\mathrm{s}}$ is resolution

\begin{tabular}{lllcll}
\hline & $R_{\mathrm{t}}\left(\mathrm{C}_{60}\right)$ & $R_{\mathrm{t}}(2)$ & Run time & $k^{\prime}(2)$ & $R_{\mathrm{S}}{ }^{a}$ \\
\hline Col 1 & 9.9 & 8.3 & 13 & 1.1 & 2.2 \\
Col 2 & 5.7 & 4.8 & 9 & 1.3 & 2.4 \\
Col 3 & 5.7 & 5.0 & 9 & 0.9 & $\mathbf{1 . 7}$ \\
Col 4 & 4.2 & 3.6 & 7 & $\boldsymbol{0 . 7}$ & $\boldsymbol{N}$ \\
Col 4 & $6.5^{b}$ & $5.2^{b}$ & $10^{b}$ & $1.3^{b}$ & $\mathbf{1 . 6}^{b}$ \\
Col 5 & 3.5 & 2.9 & 7 & $\boldsymbol{0 . 2}$ & $\boldsymbol{N A}$ \\
Col 6 & 4.0 & 3.7 & 7 & $\boldsymbol{0 . 3}$ & $\boldsymbol{N A}$
\end{tabular}

${ }^{a}$ Calculated according to the closest peak. ${ }^{b} 55 \%(\mathrm{v} / \mathrm{v})$ acetonitrile in toluene was used as mobile phase. NS: not separated. The values in bold/italic are outside the ideal range.
$\left(R_{\mathrm{S}}\right)$ for 1 is quite high also for Col 4 which indicates that effective separation may be achieved if a longer column is used. Additionally, by increasing the proportion of acetonitrile amount in the mobile phase to $55 \%$ (v/v), Col 4 also showed very good base separation of the corresponding peaks, whereas Col 5 and Col 6 still did give enough separation (ESI $\dagger$ ). The chromatograms obtained using two of the best columns (Col 1 and Col 2) with $45 \%, 50 \%$ and $55 \%(\mathrm{v} / \mathrm{v})$ acetonitrile in toluene as eluent are given in Fig. 3.

The analytical method conditions were transferred to preparative scale and $\mathbf{1}$ was successfully isolated with Col 7 using 55\% (v/v) acetonitrile in toluene with $13 \mathrm{~mL} \mathrm{~min}^{-1}$ flow (Fig. 4). With this methodology, $4000 \mu \mathrm{L}$ reaction mixture
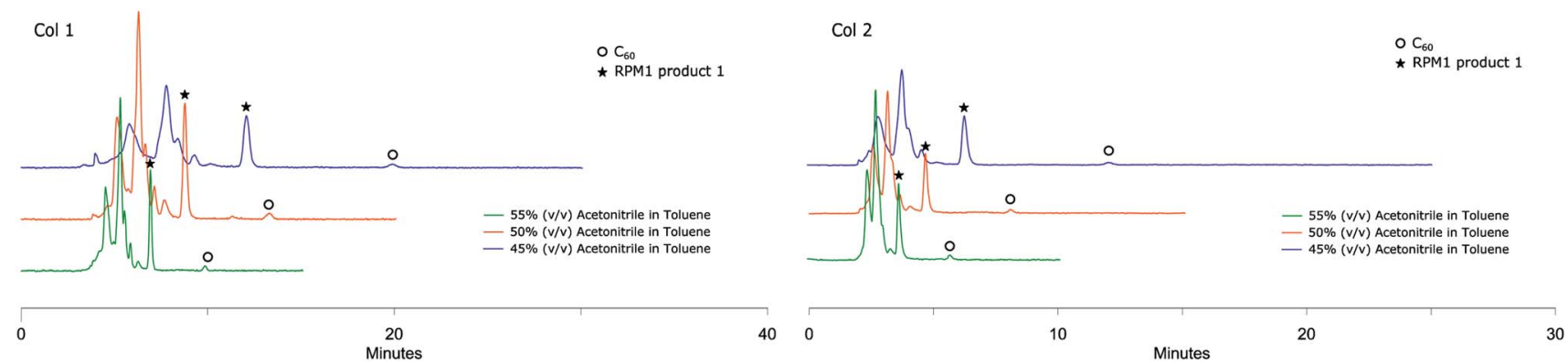

Fig. 3 RPM1 chromatograms obtained by Col 1 and Col 2 eluting with different mobile phases, detection at $285 \mathrm{~nm}$, both columns showing excellent separation of RPM1 product 1 from other components of the mixture.
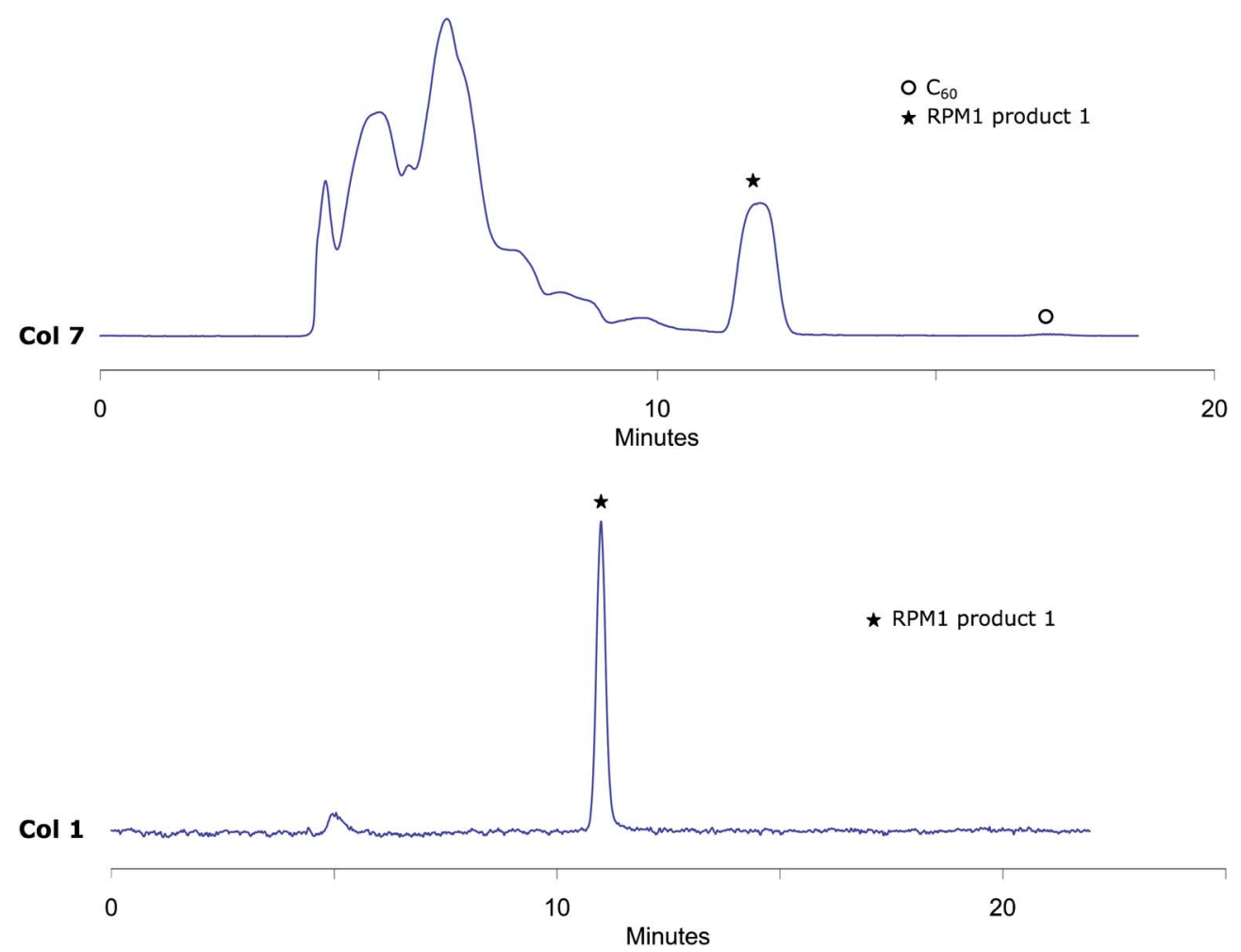

Fig. 4 Upper trace: preparative RPM1 chromatogram obtained by Col 7 eluting with $55 \%(\mathrm{v} / \mathrm{v})$ acetonitrile in toluene at $285 \mathrm{~nm}$ UV detection that shows very effective separation of RPM1 product 1 from higher adducts and unreacted $\mathrm{C}_{60}$. Lower trace: analytical chromatogram obtained by injecting collected RPM1 product 1 fraction from preparative RPM1 injection by Col 1 eluting with 55\% (v/v) acetonitrile in toluene at $285 \mathrm{~nm}$ UV detection that shows RPM1 product 1 is well separated from other peaks and isolated pure. 


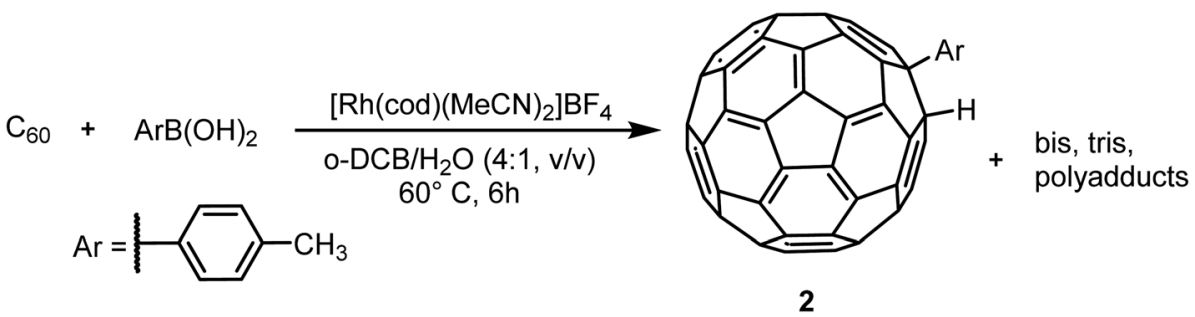

Scheme 3 Hydroarylation reaction of $\mathrm{C}_{60}$ with $p$-tolylboronic acid using a Rh catalyst. Before HPLC sample preparation, all reagents were washed away by extraction, having only unreacted $C_{60}, 2$ and higher adducts in the RPM2.

solution ( $4.8 \mathrm{mg}$ of reaction crude) was injected in each run with excellent separation of $\mathbf{1}$ from the other components in the mixture. The collected fractions belonging to RPM1 product 1 were then injected to the analytical column $\mathrm{Col} 1$, to prove that the separation had given pure fractions (Fig. 4). MALDI-MS analysis verifies that the isolated compound is RPM1 product 1 (ESI†). Our method, using standard column stationary phases, is thus not only very efficient for separation but also for isolation of components from this type of reaction mixtures.

\subsection{Analysis of reaction product mixture 2 (RPM2)}

Metal-catalyzed hydroarylation of fullerenes ${ }^{32}$ has potential to become another highly applicable wide-scope functionalization reaction for fullerene modification. The reported procedures however all suffer from poor conversion of $\mathrm{C}_{60}$ or overreaction to polyadducts. These mixtures of compounds are more challenging to separate than systems such as RPM1 due to the chemical and structural similarities of the components and reported methods rely on special stationary phases. In order to challenge this, in-house prepared hydroarylation reaction mixtures (RPM2, Scheme 3) were injected to all analytical columns using $45 \%, 50 \%$ and $55 \%(\mathrm{v} / \mathrm{v})$ acetonitrile in toluene as eluent.

The small amounts of poly-addition products were eluting faster than the mono-addition product, which in turn eluted faster than unreacted $\mathrm{C}_{60}$. Using $45 \%(\mathrm{v} / \mathrm{v})$ acetonitrile in toluene as mobile phase (Fig. 5), Col 1 and Col 2 showed excellent resolution between 2 and both unreacted $\mathrm{C}_{60}$ and polyaddition products with a short run time of 10 minutes for $\mathrm{Col} 2$ whereas $\mathrm{Col} 3$, Col 4, Col 5 and Col 6 did not separate the product well enough (Table 3). However, Resolution (Rs) for $\mathbf{2}$ is quite high also for Col 3 which indicates that effective separation may be achieved if a longer column is used. In accord with the observation for RPM1, increasing the proportion of acetonitrile to $55 \%(\mathrm{v} / \mathrm{v})$, Col 4 displayed very good base separation of the corresponding peaks whereas Col 5 and Col 6 still did not separate the components well enough (ESI†). The chromatograms obtained using two of the best columns ( $\mathrm{Col} 1$ and $\mathrm{Col} 2)$ with $45 \%, 50 \%$ and $55 \%(\mathrm{v} / \mathrm{v})$ acetonitrile in toluene as eluent are given in Fig. 6.

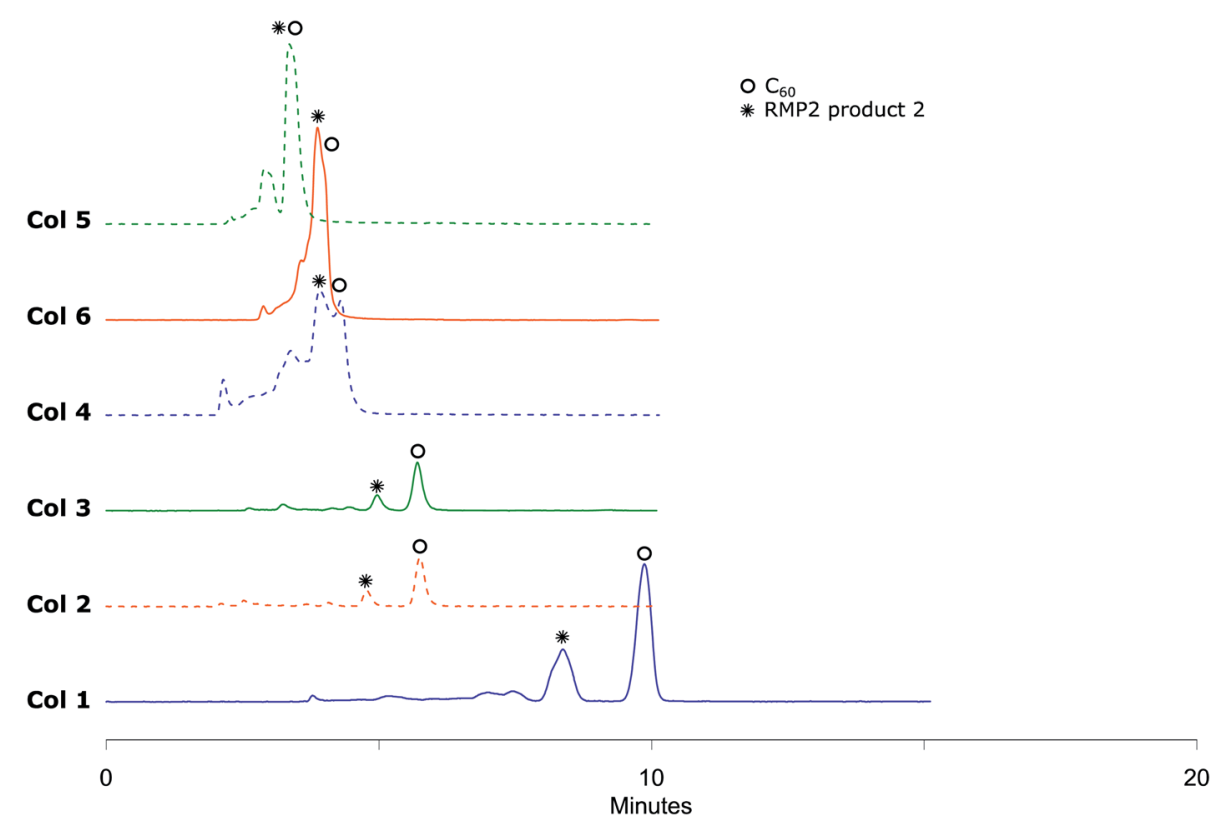

Fig. 5 RPM2 chromatograms obtained by eluting with 45\% (v/v) acetonitrile in toluene at $285 \mathrm{~nm}$ UV detection where Col 1, Col 2 and Col 3 show very good separation in short analysis time, Col 4, Col 5 and Col 6 does not separate RPM2 product 2, neither from bis, tris and polyadducts nor from unreacted $\mathrm{C}_{60}$. 


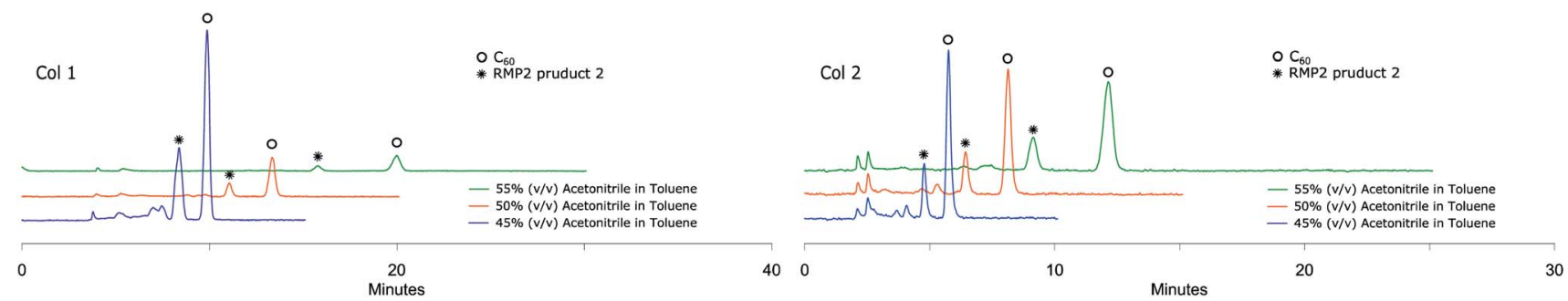

Fig. 6 RPM2 chromatograms obtained by Col 1 and Col 2 eluting with different mobile phases at $285 \mathrm{~nm}$ UV detection both showing excellent separation of RPM2 product 2.

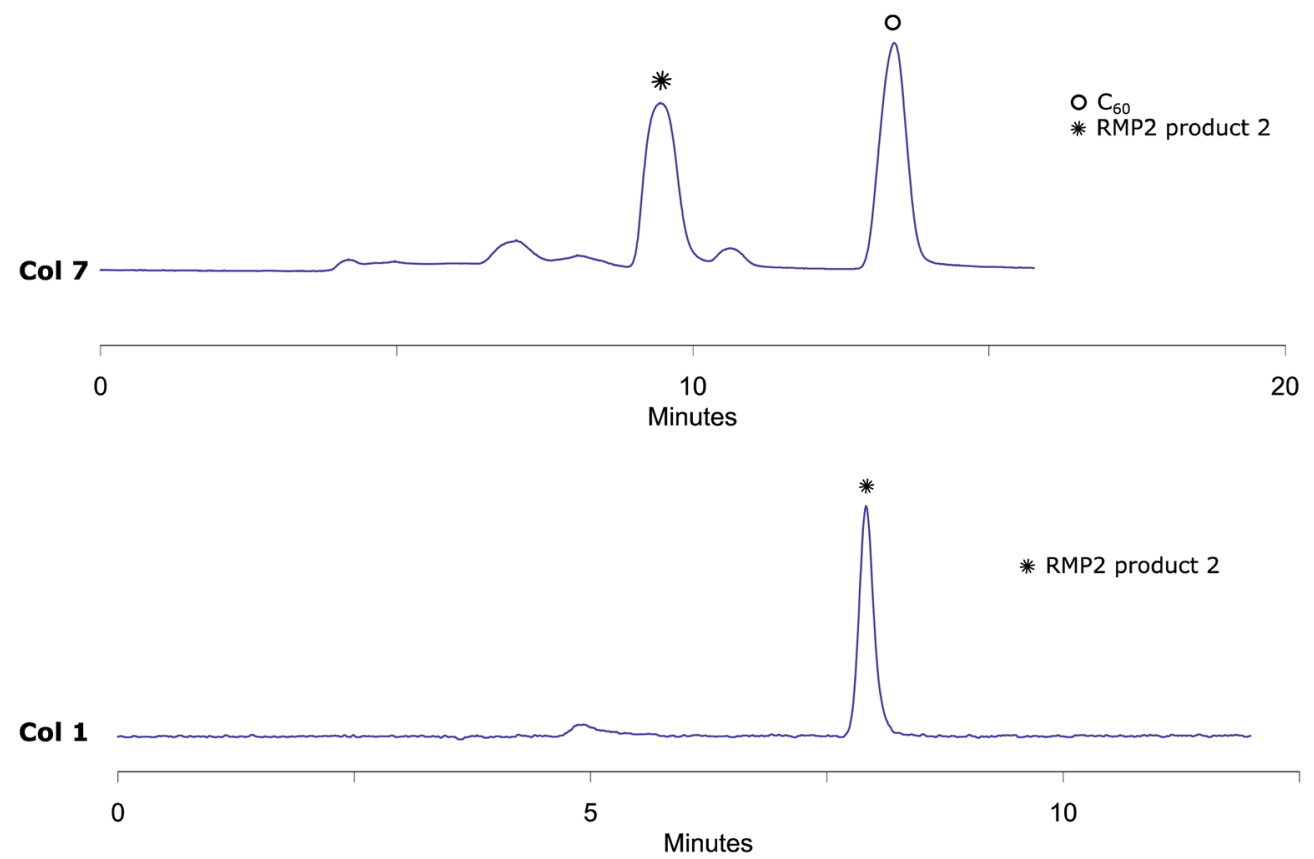

Fig. 7 Upper trace: preparative RPM2 chromatogram obtained by Col 7 eluting with $45 \%(\mathrm{v} / \mathrm{v})$ acetonitrile in toluene at $285 \mathrm{~nm}$ UV detection that shows very effective separation of RPM2 product 2 from higher adducts and unreacted $\mathrm{C}_{60}$. Lower trace: analytical chromatogram obtained by injecting collected RPM2 product 2 fraction from preparative RPM2 injection by Col 1 eluting with 45\% (v/v) acetonitrile in toluene at $285 \mathrm{~nm}$ UV detection that shows RPM2 product 2 is well separated from other peaks and isolated pure.

The analytical method conditions were transferred to preparative scale and 2 was successfully isolated with Col 7 using $45 \%(\mathrm{v} / \mathrm{v})$ acetonitrile in toluene with $10 \mathrm{~mL} \mathrm{~min}^{-1}$ flow. With this methodology, $4800 \mu \mathrm{L}$ reaction mixture solution (5.8 $\mathrm{mg}$ of reaction crude) was injected in each run with excellent separation of $\mathbf{2}$ from the other components of the mixture. The collected fractions belonging to RPM2 product 2 were then injected to the analytical column Col 1 to prove that the separation had given pure fractions (Fig. 7). MALDI-MS analysis verifies that the isolated compound is RPM2 product 2 (ESI†). Our method, using standard column stationary phases, is thus not only very efficient for separation but also for isolation of components from this type of reaction mixtures as well.

\section{Conclusions}

In this study, six analytical HPLC columns with different hydrophobic stationary phases and dimensions were tested for their separation power on a reference $\mathrm{C}_{60}+\mathrm{C}_{70}$ mixture, a fulleropyrrolidination reaction mixture and a $\mathrm{C}_{60}$ hydroarylation reaction mixture, using different proportions of acetonitrile in toluene as mobile phase. From the $\mathrm{C}_{60}+\mathrm{C}_{70}$ mixture analyses, it can be concluded that all columns can separate these molecules efficiently using $55 \%(\mathrm{v} / \mathrm{v})$ acetonitrile in toluene, however separation power decreases as the stationary phase changes from straight-chained C18 to aromatic C12. Moreover, smaller particle size for the stationary phase in a longer column results in significantly better separation. The fulleropyrrolidination reaction mixtures were excellently separated on columns with C18 stationary phases with high surface area $\left(450-400 \mathrm{~m}^{2} \mathrm{~g}^{-1}\right)$. For the $\mathrm{C}_{60}$ hydroarylation reaction mixture, in addition to $\mathrm{C} 18$, also $\mathrm{C} 12$ stationary phase with high surface area was efficient using $45 \%, 50 \%$ and $55 \%(\mathrm{v} / \mathrm{v})$ acetonitrile in toluene as eluent. The findings opens for simple and very fast analysis such as reaction conversion monitoring, product profile investigation or quantification of the components of reaction mixtures 
without the need for special column packings. Different column packings other than what is reported in this study can also be tested for separation by first injecting $\mathrm{C}_{60}$ and adjusting toluene/acetonitrile composition in the mobile phase to achieve suitable retention. Additionally, this separation method is easily applied to HPLC preparative scale for isolation of functionalized fullerene products from reaction mixtures yielding pure products in reasonably short time. The poor solubility of fullerenes in acetonitrile is the biggest challenge and diluted reaction mixture solutions $\left(1.2 \mathrm{mg} \mathrm{mL}^{-1}\right)$ were injected in large volumes to our $150 \times 21.0 \mathrm{~mm}, 5.0 \mu \mathrm{m} \mathrm{C} 18$ column to overcome this issue which resulted isolating $2-4 \mathrm{mg}$ of the product per injection. In conclusion, we have developed simple and fast HPLC methods that employ regular stationary phase columns and standard solvents; the method give efficient analytical as well as preparative separation of complex fullerene reaction mixtures.

\section{Conflicts of interest}

There are no conflicts to declare.

\section{Acknowledgements}

Financial support from $\mathrm{O}$ E Nycander Foundation and the Uppsala University KoF priority project on graphene.

\section{References}

1 D. M. Guldi and N. Martin, Fullerenes: From Synthesis to Optoelectronic Properties, Springer Netherlands, Dordrecht, 1st edn, 2002.

2 D. Chirvase, J. Parisi, J. C. Hummelen and V. Dyakonov, Nanotechnology, 2004, 15, 1317-1323.

3 M. Izquierdo, S. Osuna, S. Filippone, A. Martín-Domenech, M. Solà and N. Martín, J. Org. Chem., 2009, 74, 6253-6259.

4 H. S. Lin and Y. Matsuo, Chem. Commun., 2018, 54, 1124411259.

5 M. Yamada, R. Ochi, Y. Yamamoto, S. Okada and Y. Maeda, Org. Biomol. Chem., 2017, 15, 8499-8503.

6 L. Sánchez, M. Á. Herranz and N. Martín, J. Mater. Chem., 2005, 15, 1409-1421.

7 K. Itami, Chem. Rec., 2011, 11, 226-235.

8 B. C. Thompson and J. M. J. Fréchet, Angew. Chem., Int. Ed., 2008, 47, 58-77.

9 S. H. Park, A. Roy, S. Beaupré, S. Cho, N. Coates, J. S. Moon, D. Moses, M. Leclerc, K. Lee and A. J. Heeger, Nat. Photonics, 2009, 3, 297-303.

10 A. Mishra and P. Bäuerle, Angew. Chem., Int. Ed., 2012, 51, 2020-2067.

11 Y. Shirai, J. F. Morin, T. Sasaki, J. M. Guerrero and J. M. Tour, Chem. Soc. Rev., 2006, 35, 1043-1055.

12 N. Tagmatarchis and H. Shinohara, Mini-Rev. Med. Chem., 2001, 1, 339-348.

13 S. Jennepalli, S. G. Pyne and P. A. Keller, $R S C$ Adv., 2014, 4, 46383-46398.

14 C. Bingel, Chem. Ber., 1993, 126, 1957-1959.
15 Y. N. Biglova and A. G. Mustafin, $R S C A d v .$, 2019, 9, 2242822498.

16 F. Beuerle and A. Hirsch, Chem.-Eur. J., 2009, 15, 7434-7446.

17 J. L. Shi, X. F. Zhang, H. J. Wang, F. B. Li, X. X. Zhong, C. X. Liu, L. Liu, C. Y. Liu, H. M. Qin and Y. S. Huang, J. Org. Chem., 2016, 81, 7662-7674.

18 T. J. Kop, J. Đorđević, M. S. Bjelaković and D. R. Milić, Tetrahedron, 2017, 73, 7073-7078.

19 L. Isaacs, R. F. Haldimann and F. Diederich, Angew. Chem., Int. Ed. Engl., 1994, 33, 2339-2342.

20 R. S. Ruoff, D. S. Tse, R. Malhotra and D. C. Lorents, J. Phys. Chem., 1993, 97, 3379-3383.

21 J. L. Atwood, G. A. Koutsantonis and C. L. Raston, Nature, 1994, 368, 229-231.

22 Y. Shoji, K. Tashiro and T. Aida, J. Am. Chem. Soc., 2004, 126, 6570-6571.

23 Y. D. Yang and H. Y. Gong, Chem. Commun., 2019, 55, 37013704.

24 R. Taylor, J. P. Hare, A. K. Abdul-Sada and H. W. Kroto, J. Chem. Soc., Chem. Commun., 1990, 74, 551-558.

25 M. Santella, V. Mazzanti, M. Jevric, C. R. Parker, S. L. Broman, A. D. Bond and M. B. Nielsen, J. Org. Chem., 2012, 77, 8922-8932.

26 M. Zhang, H. J. Wang, F. B. Li, X. X. Zhong, Y. S. Huang, L. Liu, C. Y. Liu, A. M. Asiri and K. A. Alamry, J. Org. Chem., 2017, 82, 8617-8627.

27 Y. F. Li, D. Zhang, H. J. Wang, F. B. Li, L. Sun, L. Liu, C. Y. Liu, A. M. Asiri and K. A. Alamry, J. Org. Chem., 2019, 84, 2922-2932.

28 K. Kimata, K. Hosoya, T. Araki and N. Tanaka, J. Org. Chem., 1993, 58, 282-283.

29 T. Hirose, K. Moriuchi, K. Hosoya, T. Araki, N. Tanaka and K. Kimata, Anal. Chem., 1995, 67, 2556-2561.

30 S. Selmani, M. Yue Shen and D. J. Schipper, RSC Adv., 2017, 7, 19026-19029.

31 M. Maggini, G. Scorrano and M. Prato, J. Am. Chem. Soc., 1993, 115, 9798-9799.

32 M. Nambo, R. Noyori and K. Itami, J. Am. Chem. Soc., 2007, 129, 8080-8081.

33 F. Diederich, R. Ettl, Y. Rubin, R. L. Whenren, R. Beck, M. Alvarez, S. Anz, D. Sensharma, F. Wudl, K. C. Khemani and A. Koch, Science, 1991, 252, 548-551.

34 F. Diederich, R. L. Whetren, C. Thilgen, R. Etrl, I. T. O. Chao and M. M. Alvarez, Science, 1991, 254, 1768-1771.

35 A. Carboni, E. Emke, J. R. Parsons, K. Kalbitz and P. de Voogt, Anal. Chim. Acta, 2014, 807, 159-165.

36 K. Komatsu, Y. Murata, N. Takimoto, S. Mori, N. Sugita and T. S. M. Wan, J. Org. Chem., 1994, 59, 6101-6102.

37 S. Mori, M. Nambo, L. C. Chi, J. Bouffard and K. Itami, Org. Lett., 2008, 10, 4609-4612.

38 A. Astefanei, O. Núñez and M. T. Galceran, J. Chromatogr. A, 2014, 1365, 61-71.

39 A. Kolkman, E. Emke, P. S. Bäuerlein, A. Carboni, D. T. Tran, T. L. Ter Laak, A. P. Van Wezel and P. De Voogt, Anal. Chem., 2013, 85, 5867-5874. 